\title{
Investigation of the Role of Training Health Volunteers in Promoting Pap Smear Test Use among Iranian Women Based on the Protection Motivation Theory
}

\author{
Leila Ghahremani $^{1 *}$, Zahra Khiyali Harami ${ }^{1}$, Mohammad Hossein Kaveh ${ }^{1}$, \\ Sareh Keshavarzi
}

\begin{abstract}
Background: Cervical cancer is known as one of the most prevalent types of cancers and a major public health problem in developing countries which can be detected by Pap test, prevented, and treated. Despite the effective role of Pap test in decreasing the incidence and mortality due to cervical cancer, it is still one the most common causes of cancer-related deaths among women, especially in developing countries. Thus, this study aimed to examine the effect of educational interventions implemented by health volunteers based on protection motivation theory (PMT) on promoting Pap test use among women. Materials and Methods: This quasi-experimental study was conducted on 60 health volunteers and 420 women. The study participants were divided into an intervention and a control group. Data were collected using a valid self-reported questionnaire including demographic variables and PMT constructs which was completed by both groups before and 2 months after the intervention. Then, the data were entered into the SPSS statistical software, version 19 and were analyzed using Chi-square test, independent $T$-test, and descriptive statistical methods. $P<0.05$ was considered as statistically significant. Results: The findings of this study showed that the mean scores of PMT constructs (i.e. perceived vulnerability, perceived severity, fear, response-costs, self-efficacy, and intention) increased in the intervention group after the intervention $(P<0.001)$. However, no significant difference was found between the two groups regarding response efficacy after the intervention $(P=0.06)$. The rate of Pap test use also increased by about $62.9 \%$ among the study women. Conclusions: This study showed a significant positive relationship between PMT-based training and Pap test use. The results also revealed the successful contribution of health volunteers to training cervical cancer screening. Thus, training interventions based on PMT are suggested to be designed and implemented and health volunteers are recommended to be employed for educational purposes and promoting the community's, especially women's, health.
\end{abstract}

Keywords: Health volunteer - cervical cancer - Pap smear test - protection motivation theory

Asian Pac J Cancer Prev, 17 (3), 1157-1162

\section{Introduction}

Cervical cancer is the second most common cancer among women worldwide (Asonganyi et al., 2013) and more than $85 \%$ of the cases have reported in developing countries (Jemal et al., 2011). According to World Health Organization (WHO), $25 \%$ of deaths among women are caused by malignant tumors, $18 \%$ of which being associated with cervical cancer (Yakhforoshha et al., 2008). Epidemiological and statistical surveys have indicated that cervical cancer is the fourth common cancer among Iranian women (Jowzi et al., 2013) and its incidence rate was reported to be $7.1 \%$ in different provinces (Jalilian et al., 2011). Cervical cancer is one of the most preventable cancers due to its long precancerous period, availability of effective screening, and treatment of primary lesions (Logan et al., 2011). Thus, screening programs, such as Pap smear test, play an important role in early detection, or prevention or delay in progression of cervical abnormalities to invasive cancer (Behnamfar and Azadehrah, 2015). PAP smear test, used in early diagnosis, is an easily performed, low-cost, harmless test with a high sensitivity and it also decreases treatment burden, morbidity and mortality (Ozdemir and Bilgili, 2010). The advantageous application of Pap test in detecting cervical cancer has been shown in countries, such as Sweden and Finland, where national screening programs have been implemented. The prevalence, implications, and mortality rate of cervical cancer are dramatically low in these countries (Hyacinth et al., 2012). However, in Iran the number of women seeking for screening is limited due to the limited screening programs and lack of information about such programs. Hence, most cases with cervical cancer are detected at late stages (Rouhollahi et al., 


\section{Leila Ghahremani et al}

2014; Behnamfar and Azadehrah, 2015). Cervical cancer preventive programs can be effective in increasing cervical cancer knowledge, perceived susceptibility, and cancer prevention behavior (Choi, 2013). Yet, Pap test use can be enhanced among women through increasing knowledge of cervical cancer screening (Aswathy et al., 2012), implementing educational interventions (Park et al., 2005), health education programs (Ranabhat et al., 2014) and improving provision of care services (Ghahramaninasab et al., 2014). Nevertheless, free screening services cannot solely prevent cervical aggressive lesions and appropriate training regarding application of these methods is vital (Ghahramaninasab et al., 2014). This necessitates execution of educational interventions. One training method in this respect is recruiting health volunteers to train women (Miri et al., 2012; Abuidris et al., 2013; Kaveh et al., 2014). In this way, health volunteers who are socially accepted among women in a neighborhood, have spare time, and are motivated (Lauber et al., 2002) are employed to improve and delivery of healthcare services and help individuals in the community to live healthier (Naylor et al., 2013). Also, due to cultural, economic, and social similarities and common beliefs, health volunteers can play an effective role in implementation of educational programs and empowerment of society members, especially women (Abuidris et al., 2013; Kaveh et al., 2013). Moreover, a number of studies have shown that the most efficient training programs are based on the theory-based methods originated from behavior change models. The first step to design an educational program is selecting an appropriate and effective theory or model (Jalilian et al., 2011). In this regard, the constructs of Protection Motivation Theory (PMT) are highly important in predicting cancer prevention behaviors (Jowzi et al., 2013) and describing screening behaviors, such as cervical screening (Hassani et al., 2014). PMT was first developed by Rogers (1975) and has been broadly accepted and used as a framework for predicting health behaviors and implementing health-related interventions (Milne et al., 2000). This theory was developed to address the effects of health risk phobia (cervical cancer) on health attitudes and behaviors as well as the positive effects of the evoked fear on behaviors. Rogers suggested that fear could positively affect protection motivation (intentions) according to five constructs, namely perceived vulnerability, perceived severity, response costs, response efficacy, and selfefficacy (Jowzi et al., 2013). This theory has been used in few studies, such as those by Fry and Prentice-Dunn, 2006; Baghianimoghadam et al., 2011; Dehdari et al., 2014; Ghahremani et al., 2014. For instance, Fry and PrenticeDunn demonstrated that developing interventions based on PMT could encourage women to perform breast selfexamination (Fry and Prentice-Dunn, 2006). Dehdari et al. also confirmed the effect of this theory on encouraging women to take Pap smear test (Dehdari et al, 2014).

Therefore, the present study aims to evaluate the role of health volunteers in promoting cervical screening behaviors based on PMT among women in Fasa, Fars province, Iran in 2014.

\section{Materials and Methods}

This quasi-experimental study was conducted on health volunteers and the women under their coverage in 6 health stations in Fasa. The subjects were selected through multi-stage sampling. At the first stage, based on the previous studies, the mean difference formula, $\alpha=0.05$, and power of $80 \%$ and using power SSC software, a 50-subject sample size was determined for the study (25 subjects in each group). However, considering the loss rate of $20 \%$, the sample size was increased to 60 (30 subjects in each group). According to the health volunteers protocol, each volunteer usually covers 50 households. Among the women covered by each health volunteer, 7 ones (a total of 420 participants) who were married, were not pregnant, had never experienced Pap test, did not have cervical cancer, had no history of hysterectomy operations, and were willing to participate in the study were selected.

The study data were collected using a questionnaire which was completed by the women under study. The questionnaire consisted of demographic information as well as 26 questions on the constructs of PMT (perceived vulnerability ( 3 items), perceived severity (4 items), response efficacy (4 items), intention (3 items), fear (3 items), response costs (2 items), and self-efficacy ( 7 items)). The first four items were measured using a Likert scale ranging from 1 (completely disagree) to 5 (completely agree).Besides, fear and response costs were evaluated through a Likert scale ranging from 1 (completely agree) to 5 (completely disagree). Additionally, self-efficacy regarding performance of Pap test was assessed on a Likert scale ranging from 1 (completely unconfident) to 5 (completely confident). The score of each construct was computed by summing up the scores of its subscales. This questionnaire was developed and validated by Hasani et al. (content validity: $89 \%$ ). In addition, its reliability was confirmed by Cronbach's alpha of 0.93-0.70 and internal correlation coefficient of 0.72-0.96. Moreover, exploratory

Table 1. Comparative Evaluation of Demographic Variables among Women

\begin{tabular}{lcrr}
\hline & $\begin{array}{c}\text { Intervention } \\
\text { group } \\
(\mathrm{n}=210)\end{array}$ & $\begin{array}{c}\text { Control } \\
\text { group } \\
(\mathrm{n}=210)\end{array}$ & $\begin{array}{c}\text { Chi- } \\
\text { square } \\
\text { test }\end{array}$ \\
\hline $\begin{array}{l}\text { Marital Status } \\
\text { Married }\end{array}$ & $\begin{array}{c}\text { 201(95.7\%) } \\
\text { Died }\end{array}$ & $\begin{array}{r}197(93.8 \%) \\
13(6.2 \%)\end{array}$ & 0.38 \\
Education Level & $9(4.3 \%)$ & & 0.31 \\
$\quad$ Illitrate & $12(5.7 \%)$ & $7(3.3 \%)$ & \\
$\leq 12^{\text {th }}$ (grade) & $159(75.7 \%)$ & $155(73.8 \%)$ & \\
$>12^{\text {th }}$ (grade) & $39(18.6 \%)$ & $48(22.9 \%)$ & \\
Job & & & 0.71 \\
Housewife & $195(92.9 \%)$ & $193(91.9 \%)$ & \\
$\quad$ Employee & $15(7.1 \%)$ & $17(8.1 \%)$ & \\
Economic status & & & 0.65 \\
$\quad$ Weak & $19(9 \%)$ & $14(6.7 \%)$ & \\
Moderate & $53(25.2 \%)$ & $53(25.2 \%)$ & \\
$\quad$ Good & $138(65.7 \%)$ & $143(68.1 \%)$ & \\
Family History of Cancer & & 0.82 \\
$\quad$ Yes & $51(24.3 \%)$ & $53(25.2 \%)$ & \\
No & $159(75.7 \%)$ & $157(74.8 \%)$ & \\
\hline
\end{tabular}


Role of Training Health Volunteers in Promoting Pap Smear Test Use among Women Based on Protection Motivation Theory Table 2. Comparison of the Mean Scores of PMT Constructs in the Intervention and Control Groups before and after the Intervention

\begin{tabular}{|c|c|c|c|c|c|c|}
\hline & \multicolumn{3}{|c|}{ Pre-intervention } & \multicolumn{3}{|c|}{ Tow-months after intervention } \\
\hline & Intervention group & Control group & P-Value & Intervention group & Control group & P-Value \\
\hline Perceived vulnerability & $7.91 \pm 2.28$ & $7.84 \pm 1.90$ & 0.72 & $9.43 \pm 2.56$ & $7.93 \pm 2.41$ & $\mathrm{p}<0.001$ \\
\hline Perceived severity & $13.19 \pm 2.89$ & $13.35 \pm 2.53$ & 0.54 & $15.32 \pm 3.07$ & $13.50 \pm 2.69$ & $\mathrm{p}<0.001$ \\
\hline Fear & $8.10 \pm 2.67$ & $8.80 \pm 2.76$ & 0.009 & $10.23 \pm 2.62$ & $8.65 \pm 2.67$ & $\mathrm{p}<0.001$ \\
\hline Response-costs & $5.72 \pm 1.92$ & $5.73 \pm 1.99$ & 0.96 & $6.89 \pm 2.08$ & $5.56 \pm 2.25$ & $\mathrm{p}<0.001$ \\
\hline Response efficacy & $16.38 \pm 2.56$ & $15.50 \pm 3.10$ & 0.002 & $16.41 \pm 2.92$ & $15.87 \pm 3.22$ & $\mathrm{p}=0.06$ \\
\hline Self-efficacy & $26.99 \pm 5.29$ & $26.54 \pm 5.70$ & 0.41 & $29.44 \pm 5.07$ & $26.52 \pm 5.66$ & $\mathrm{p}<0.001$ \\
\hline Intention & $11.96 \pm 2.45$ & $11.52 \pm 2.70$ & 0.08 & $13.06 \pm 2.25$ & $11.47 \pm 2.62$ & $\mathrm{p}<0.001$ \\
\hline
\end{tabular}

Table 3. The rate of Pap test use in the intervention $(n=210)$ and control $(n=210)$ groups after the intervention

\begin{tabular}{lcccccc}
\hline & \multicolumn{2}{c}{ Tow-months after intervention } & & \multicolumn{2}{c}{ Pre-intervention } & \\
\cline { 2 - 3 } & Intervention group & Control group & & Intervention group & Control group & P-Value \\
\hline Pap test practice & $0(0 \%)$ & $0(0 \%)$ & & $132(62.85 \%)$ & $12(5.71 \%)$ & $\mathrm{p}<0.001$ \\
\hline
\end{tabular}

analysis was 72.76 for all the 7 parameters and adaptive analysis showed appropriateness of the items (Hassani et al., 2014).

Training classes were conducted for the health volunteers through three 120-minute sessions using PowerPoint slide show and educational pamphlets and booklets. The educational content of the training sessions included introduction to cervical cancer, signs and risk factors, complications and their effects on daily life, importance of Pap test in early detection and treatment of cervical cancer, method of sampling, and places for performing Pap test. Then, the health volunteers in the intervention group trained the women under their coverage face-to-face using pamphlets for 3 sessions over 21 days. The data were collected before and 2 months after the intervention. It should be noted that this study aimed at promotion of performance of Pap test among women; thus, the women were required to provide their Pap test results or the date and location of taking the Pap test. After all, the data were entered into the SPSS statistical software, version 19 and were analyzed using independent T-test, Chi-square test. $\mathrm{P}<0.05$ was considered as statistically significant.

\section{Results}

The mean age of the health volunteers was $46.73 \pm 7.21$ and $43.96 \pm 7.14$ years in the intervention and the control group, respectively. Besides, $96.7 \%$ of the subjects in the intervention group and $93.3 \%$ of those in the control group were married and the rest were single. In addition, the majority of the health volunteers had below diploma degrees $(60 \%$ in the intervention and $60 \%$ in the control group) and all of them were homemakers. The results of Chi-square test revealed no significant difference between the two groups regarding the demographic variables.

The mean age of the women in the intervention and control groups was $38 \pm 9.93$ and $37.09 \pm 9.85$ years, respectively. Additionally, the women's mean age at marriage was $20.14 \pm 4.16$ and $20.48 \pm 4.91$ years in the intervention and the control group, respectively. Besides, the mean number of pregnancies was $2.50 \pm 1.50$ in the intervention group and $2.56 \pm 1.67$ in the control group. The results of Chi-square test revealed no significant difference between the two groups regarding the demographic variables (Table 1).

According to Table 2, a significant difference was observed between the two groups regarding the mean scores of PMT constructs(perceived vulnerability, perceived severity, fear, cost-response, efficacy, and intention) after the intervention. However, no significant difference was found between the two groups regarding response efficacy after the intervention.

At the beginning of the study, none of the study participants had experienced Pap test. After the intervention, however, $62.85 \%$ of the participants in the intervention group and $5.71 \%$ of those in the control group underwent Pap test. The results have been presented in Table 3 .

\section{Discussion}

The findings of the present study showed a significant increase in the mean score of perceived vulnerability in the intervention group after the intervention, indicating the effect of the educational program on PMT constructs. Dehdari, Karimi, Pirzadeh, Yakhforoshha, and Latifa also reported improvement of perceived vulnerability after the training intervention in the intervention group (Yakhforoshha et al., 2008; Karimi et al., 2012; Pirzadeh et al., 2012; Latifa et al., 2013; Dehdari et al., 2014). Besides, Shekhar revealed that almost half of the nurses who had never experienced Pap test believed that they were not vulnerable to cervical cancer (Shekhar et al., 2013). Moreover, Hoque conducted a study on South African students and Demirtas evaluated Turkish women and demonstrated that the limited number of Pap tests was due to lack of perceived vulnerability (Hoque et al., 2009; Demirtas and Acikgoz, 2013). However, Park 
stated that there was no significant relationship between perceived vulnerability and Pap test use among women in intervention and control groups (Park et al., 2005). This might be due to the fact that the intervention was not long enough to result in changes in the participants' attitudes. These results demonstrate that the more vulnerable individuals consider themselves to health risks, such as cervical cancer, the more their intention to take Pap test will be.

The results of the present study revealed a significant increase in the intervention group's mean score of perceived severity compared to the control group after the intervention. These results were in accordance with those obtained by Yakhforoshha, Pirzadeh, Karimi, Peter, in a study on women in Ghana, and Latifa in a study on Egyptian women (Peter et al., 2006; Yakhforoshha et al., 2008; Karimi et al., 2012; Pirzadeh et al., 2012; Latifa et al., 2013). Correspondingly, Holmes proposed that perceived severity related to breast cancer risk predicted the intention to take genetic tests for detecting breast cancer (Holmes et al., 2002). Jalilian also introduced perceived severity as the most important predictor of Pap test use (Jalilian et al., 2011). Nevertheless, these results were in contrast to those of the studies by Dehdari, Demirtas, and Fang (Fang et al., 2007; Demirtas and Acikgoz, 2013; Dehdari et al., 2014). These differences might be attributed to women's low perception of cervical cancer risks and the related complications. This implies that in case a person efficiently perceives the risks and consequences of the disease, one will show more preventive behaviors.

The findings of the current study showed a significant increase in the mean score of fear in the intervention group compared to the control group after the intervention. Baghianimoghadam also found a significant relationship between fear and skin cancer preventive behaviors (Baghianimoghadam et al., 2011). However, Dehdari found no significant relationship between fear and Pap test use among women (Dehdari et al., 2014). In our study, the training intervention was implemented by health volunteers. This could indirectly create higher levels of fear among women, eventually increasing their motivation to show preventive behaviors against cervical cancer.

Considering the mean response costs, the present study results indicated a significant increase in the intervention group in comparison to the control group after the intervention. Holmes conducted a study entitled "Application of protection motivation theory for genetic testing to detect breast cancer" and reported that perceived costs could enhance motivation to perform genetic tests (Holmes et al., 2002), which is consistent with the findings of the present study. These results suggest that women are more likely to perform screening costs if they find the costs of such operations logical and acceptable.

The present study findings showed no significant difference between the intervention and control groups regarding the mean score of response efficacy, which is similar to the results of the study by Dehdari et al. (2014). However, these results were on the contrary to those gained by Ghahremani et al. (2014), which might result from the short period of the intervention for changing the women's attitude towards Pap test. In fact, the women under the present investigation considered Pap test as an instrument for diagnosis of cervical cancer rather than a screening tool.

Our study results indicated a significant increase in the intervention group's self-efficacy scores compared to the control group after the intervention. This was in agreement with the results obtained by Dehdari, Karimi, and Ghahremani (Karimi et al., 2012; Dehdari et al., 2014; Ghahremani et al., 2014). Fang and Park also demonstrated a positive relationship between higher self-efficacy and Pap test use, such a way that improved self-efficacy increased Pap test use (Park et al., 2005; Fang et al., 2007). Similar results were also obtained by Hoque among University Students in South Africa (Hoque et al., 2014). Bandura and Adams proposed self-efficacy as the most important predictor of behavioral changes. They reported that self-efficacy positively influenced health behaviors, such a way that individuals with low selfefficacy were less likely to conduct new health behaviors (Karimi et al., 2012).

In the present study, the intervention group was more intended to perform Pap test after the intervention, showing the positive effects of the educational intervention on promoting Pap test use among women as well as the health volunteers' contribution to training these individuals. Dehdari and Park also confirmed these findings (Park S et al., 2005; Dehdari et al., 2014).

This study also suggested an increase in Pap test use among the intervention group participants. Before the intervention, the women in both groups had never experienced Pap test. After the intervention, however, the rate of Pap test use increased to $62.85 \%$ in the intervention group, indicating the positive effect of the educational intervention on Pap test use among women. Dehdari, Pirzadeh and Karimi also reported an increase in Pap test use in the intervention groups (Karimi et al., 2012; Pirzadeh et al., 2012; Dehdari et al., 2014). Similarly, Abiodun conducted a study in Nigeria and reported that the rate of Pap test use increased among women after training courses using media, such as video tapes (Abiodun et al., 2014). In our study, 12 cases $(5.71 \%)$ in the control group also performed Pap test after the intervention, which might have resulted from their sensitivity after completing the study questionnaire.

In some countries, such as Iran, health volunteers are considered as one of the major social human resources who are able to meet many social health needs by actively assisting the health systems and health entities. Since the majority of health volunteers are woman, they can considerably meet women's health requirements and play an important role in empowerment of women (Behdjat et al., 2009).

The findings of the current study proved the positive role of health volunteers in promoting Pap test use among women. These findings were in line with those of the study by Abuidris conducted in Sudan and Kaveh in Iran (Abuidris et al., 2013; Kaveh et al., 2014). Similarly, Miri in a quasi-experimental study showed that health volunteers positively influenced the women's healthrelated attitudes and knowledge (Miri et al., 2012).

This study had some limitations, the first of which 
Role of Training Health Volunteers in Promoting Pap Smear Test Use among Women Based on Protection Motivation Theory

being data collection using a questionnaire because some participants were likely not to provide correct answers. The short intervention and follow-up periods was another limitation of the present study. In order to evaluate the effects of training on the subjects' performance, they are suggested to be followed up until six months after the intervention. One other study limitation was women's unwillingness to undergo Pap test due to its high costs. Moreover, this study was conducted on the women covered by urban health centers. Thus, PMTbased educational interventions are recommended to be performed for other community members, such as students, teachers, and villagers. Since men are great contributors to women's health, training programs must be developed for men, as well.

The findings of this study showed the effectiveness of the educational intervention based on PMT on Pap test use among the women and as well as the great contribution of health volunteers to training these individuals. Hence, health administrators are suggested to focus on the key role of health volunteers as the most efficient workers in training and promoting community health and to cooperate with them to promote women's health.

\section{Acknowledgements}

This article was extracted from Zahra Khiyali Harami's M.Sc. thesis in Health Education (registration No. 7259-93) approved and financially supported by the Vice-chancellor for Research Affairs of Shiraz University of Medical Sciences. Hereby, the authors would like to acknowledge all the health volunteers and women who cooperated in the research. They are also grateful for Ms. A. Keivanshekouh at the Research Improvement of Center of Shiraz University of Medical Sciences for improving the use of English in the manuscript.

\section{References}

Abiodun O, Olu- Abiodun O, Sotunsa J, et al (2014). Impact of health education intervention on knowledge and perception of cervical cancer and cervical screening uptake among women rural communities in Nigeria. BMC Public Health, 14, 814-22.

Abuidris DO, Elsheikh A, Ali M, et al (2014). Breast-cancer screening with trained volunteers in a rural area of Sudan: a pilot study. Lancet Oncol, 14, 363-70.

Asonganyi E, Vaghasia M, Rodrigues C, et al (2013). Factors affecting compliance with clinical practice guidelines for pap smear screening among healthcare providers in africa: systematic review and meta summary of 2045 individuals. PLOS ONE, 8, 72712.

Aswathy S, Quereshi MA, Kurian B, Leelamoni K (2012). Cervical cancer screening: current knowledge and practice among women in a rural population of Kerala, India. Indian J Med Res, 136, 205-10.

Baghianimoghadam MH, Mohammadi S, Mazloomi Mahmoudabad SS, et al (2011). The effect of education based on protection-motivation theory on skin cancer preventive practices among female high school students in Yazd. ofoghe-danesh. J Gonabad University Med Sci, 17, 27-34.

Behdjat H, Rifkin SB, Tarin E, et al (2009). A new role for women health volunteers in urban islamic republic of Iran. Eastern
Mediterranean Health J, 15, 1164-73.

Behnamfar F, Azadehrah M (2015). Factors associated with delayed diagnosis of cervical cancer in Iran - a Survey in Isfahan City. Asian Pac J Cancer Prev, 16, 635-9.

Choi SY (2013). Development of an educational program to prevent cervical cancer among immigrants in Korea. Asian Pac J Cancer Prev, 14, 5345-9.

Dehdari T, Hassani L, Hajizadeh E, et al (2014). Effects of an educational intervention based on the protection motivation theory and implementation intentions on first and second pap test practice in Iran. Asian Pac J Cancer Prev, 15, 7257-61.

Demirtas B, Acikgoz I (2013). Promoting attendance at cervical cancer screening: understanding the relationship with Turkish womens' health beliefs. Asian Pac J Cancer Prev, 14, 333-40.

Fang CY, Ma GX, Tan Y, et al (2007). A multifaceted intervention to increase cervical cancer screening among underserved Korean women. Cancer Epidemiol Biomarkers Prev, 16, 1298-302.

Fouda LM, Elhossiny Elkazeh EA (2013). The impact of an educational intervention on women's knowledge and perception regarding cervical cancer and human papillomavirus vaccines in tanta city: applying health belief model. Life Sci J, 10, 997-1005.

Fry RB, Prentice-Dunn S (2006). Effects of a psychosocial intervention on breast self-examination attitudes and behaviors. Health Educ Res, 21, 287-95.

Ghahramaninasab P, Shahnazi M, khalil AF, et al (2014). Factors associated with cervical cancer screening in women referred to health centers in Tabriz. Iran J Obstetrics Gynecol Infertility, 16, 15-24.

Ghahremani L, Faryabi R, Kaveh MH (2014). Effect of health education based on the protection motivation theory on malaria preventive behaviors in rural households of Kerman, Iran. Int J Prev Med, 5, 463-71.

Hassani L, Dehdari T, Hajizadeh E, et al (2014). Development of an instrument based on the protection motivation theory to measure factors influencing women's intention to first Pap test practice. Asian Pac J Cancer Prev, 15, 1227-32.

Helmes AW (2002). Application of the protection motivation theory to genetic testing for breast cancer risk. Prev Med, 35, 453-62.

Hoque M E, Ghuman Sh, Coopoosmay R, et al (2014). Cervical cancer screening among university students in south africa: a theory based study. PLOS ONE, 9, 111557.

Hyacinth HI, Adekeye OA, Ibeh JN, Osoba T (2012). Cervical cancer and pap smear awareness and utilization of pap Smear test among federal civil servants in North Central Nigeria. PLoS ONE, 7, 46583.

Jalilian F, Mirzaei Alavijeh M, Emdadi Sh, et al (2011). Predicting factors related with pap smear results among women based on health belief model. J Health System, 7, 1226-34.

Jemal A, Bray F, Center MM, et al (2011). Global cancer statistics. CA Cancer J Clin, 61, 69-90.

Jowzi F, Hashemifard T, Morowatisharifabad M, et al (2013). Factors associated with pap smear screening test among women aged 15-49 based on protection motivation theory. $\mathrm{J}$ of School of Nursing and Midwifery. Tehran University Med Sci, 19, 29-40.

Kaveh MH , Najafi A, PourAlimohammadi N, et al (2013). Effect of a menopausal health training led by health volunteers on community women's knowledge; an interventional study. Quarterly University Health Yazd, 11, 135-49.

Karimy M, Gallali M, Niknami Sh, et al (2012). The effect of health education program based on Health Belief Model on the performance of Pap smear test among women referring 
to health care centers in Zarandieh. J Jahrom University Med Sci, 10, 53-9.

Lauber C, Nordt C, Falcato L, et al (2007). Determinants of attitude to volunteering in psychiatry: results of a public opinion survey in Switzerland. Int J Soc Psychiatry, 48, 209-19.

Logan L, Mcilfatick S (2011). Exploring women's knowledge, experiences and perceptions of cervical cancer screening in an area of social deprivation European. J Cancer Care, 20, 720-7.

Milne S, Sheeran P, Orbell SH (2000). Prediction and intervention in health-related behavior: a meta-analytic review of protection motivation theory. J Appl Soc Psych, 30, 106-43.

Miri MR, Ramazani AA, Moodi M, et al (2012). The effects of suburban villages' health volunteer plan on women's health knowledge and attitude. J Educ Health Promot, 1, 12.

Naylor C, Mundle C, Weaks L, et al (2013). Volunteering in health and care Securing a sustainable future. King's Fund, $1-43$.

Ozdemir O, Bilgili N (2010). Knowledge and practices of nurses working in an education hospital on early diagnosis of breast and cervix cancers. Prev Med Bull, 9, 605-12.

Park S, Chang S, Chung C (2005). Effects of a cognitionemotion focused program to increase public participation in Papanicolaou smear screening. Public Health Nurs, 22, 289-98.

Peter N, Abotchie M, Navkiran KSh (2009). Cervical cancer screening among college students in Ghana: knowledge and health beliefs. Int J Gynecol Cancer, 19, 412-6.

Pirzadeh A, Mazaheri MA (2012). The effect of education on women's practice based on the health belief model about pap smear test. Int J Prev Med, 3, 585-90.

Ranabhat S, Tiwari M, Dhungana G, et al (2014). Association of knowledge, attitude and demographic variables with cervical pap smear practice in Nepal. Asian Pac J Cancer Prev, 15, 8905-10.

Rouhollahi MR, Mohagheghi MA, Mohammadrezai N, et al (2014). Situation analysis of the national comprehensive cancer control program (2013) in the I. R. of Iran; Assessment and recommendations based on the IAEA impact mission. Arch Iran Med, 17, 222-31.

Shekhar S, Sharma C, Thakur S, et al (2013). Cervical cancer screening: knowledge, attitude and practices among nursing staff in a tertiary level teaching institution of rural India. Asian Pac J Cancer Prev, 14, 3641-5.

Yakhforushha A, Solhi M, Ebadi Fard Azar F (2009). Effects of education via health belief model on knowledge and attitude of voluntary health workers regarding Pap smear in urban centers of Qazvin. $J$ Nursing and Midwifery martyr Beheshti, 62, 24-9. 